Aus der Nervenabteilung der medizinischen Klinik Heidelberg (Leiter: Prof. Dr. J. Hoffmann).

\title{
Über einen Fall von Aneurysma der Arteria vertebralis sinistra.
}

Von

Dr. Steckelmacher,

Assistenzarzt.

(Mit 3 Abbildungen.)

Griesinger ') veröffentlichte 1862 einen Fall von diffuser Erweiterung der Art. basilaris und vertebralis, der ausser Pupillenveränderungen, starker Benommenheit und Zuckungen im rechten Arm keine weiteren Symptome zeigte; die Diagnose war zu Lebzeiten nicht gestellt worden. Er erwähnt in derselben Arbeit, in der er 20 Fälle aus der Literatur zusammengestellt hat, einen ähnlichen von Guéniot beschriebenen Fall. Eine gleichfalls zusammenfassende Arbeit von Lebert aus dem Jahre 1866 ergab, dass die Aneurysmen der. Art. vertebralis sehr selten sind. Lebert, der keine eigenen Fälle beibringt, betont, dass die Dickendifferenz, die zwischen den beiden Vertebralarterien bestehe, die Aneurysmenbildung der linksseitigen Vertebralarterie begünstige, denn letztere sei, weil von Natur weiter, dem Druck des aus dem linken Ventrikel ausgeworfenen Blutes mehr ausgesetzt als die rechte Vertebralis.

Unter den von $0 p e^{2}$ ) im Jahre 1892 zusammengestellten Fällen von Aneurysmen der Art. vertebralis sind 6 linksseitige und ein rechtsseitiges, darunter die 3 von $\mathrm{Möser}{ }^{3}$ ) und einer von Friedrich Schultze ${ }^{4}$ ). Sodann besehrieben Oppenheim und Siemerling ${ }^{5}$ ) einen Fall, der unter dem Bilde der apoplektiformen Bulbärparalyse verlaufen war.

E. von $\mathrm{Hoffmann}^{6}$ ) betont die relative Häufigkeit dieser. Aneurysmen. Er fand unter 73 Gehirnaneurysmen 7, die von der Art. vertebralis sin. und 3, die von der Vertebralis dextr. ausgegangen waren.

$\mathrm{Zu}$ Lebzeiten hatten sie Symptome von intrakranieller Blutung geboten, deren Ursache erst durch die Obduktion aufgedeckt worden war. 
Möser hebt in seiner Arbeit als wichtig und entscheidend für die Differentialdiagnose von anderen basalen Affektionen des Gehirns folgende 3 Punkte hervor:

1. allgemeine Kreislaufverhältnisse (Atherom, luetische Gefässerkrankung).

2. Gefässgeräusche zwischen Proc. mastoideus und Wirbelsäule.

3. Fehlen von Stauungspapille. Letzteres Moment soll gegen die Möglichkeit des basalen Tumors sprechen.

Wie ersichtlich, ist die Diagnose des Aneurysma der Art. vertebralis am Krankenbett ganz selten mit Sicherheit gestellt worden. Um so mehr mag es von Interesse sein, einen typischen za Lebzeiten diagnostizierbaren Fall kennen zu lernen, der alle Symptome bietet, die man von einem Aneurysma der Art. vertebralis theoretisch fordern kann.

Es handelt sich um einen 46 jährigen Metzger, dessen Familienanamnese belanglos ist. Er erinnert sich keiner Kinderkrankheiten, war auch später nie krank. Seit 16 Jahren verheiratet, Ehefrau und 4 Kinder gesund. Die Frau hatte keine Fehlgeburten. Venerische Infektion negiert. 4-5 Zigarren pro Tag. 1/2 Liter Wein täglich. Im Januar 1913 bekam er Schmerzen in der Nackengegend, die nach abwärts zwischen die Schulterblätter, aufwärts in den ganzen Kopf, besonders in die Scheitelgegend ausstrahlten. Gleichzeitig sei der Nacken stark angeschwollen und er habe Schluckbeschwerden gehabt. Die Schmerzen exazerbierten bei Kopfbewegung; so konnte er sich im Bett nur aufrichten, wenn er den Kopf dabei festhielt. Bettlägerig war er nicht; ob er Fieber hatte, kann er nicht angeben. Die Schluckstörungen dauerten nur wenige Tage, die Sclmerzen im Nacken ca. 4 Wochen; Schwitzen brachte Erleichterung. Zu gleicher Zeit stellte sich Sprachstörung ein, er stotterte angeblich und fürchtete, die Sprache zu verlieren; auch soll die Zunge beim Vorstrecken nach links abgewichen sein und linkshälftig gezuckt haben. Ferner sei die linke Wange wie tot "lahm" geworden, der linke Mundwinkel habe herabgehangen, so dass er vorsichtig trinken musste, damit nicht die Flüssigkeit zum Munde herauslief; gleichzeitig fühlte er Schmerzen in der linken Wange. Das Kauen machte keine Schwierigkeit. Er meint, heiser gewesen zu sein, kann dies aber nicht mit Sicherheit angeben.

Die Halswirbelsäule wurde im ersten halben Jahr ganz steif gehalten, dann stellte sich langsam Schiefhaltung des Kopfes nach links ein. In der ersten Zeit taumelte er auch beim Gehen, er konnte dabei die Richtung nicht ganz einhalten; fürchtete, auf der Strasse an Passanten anzustossen. Bald nach Beginn der Schmerzen hörte er anf dem linken Ohr schlechter, hatte dauernd darin ein Blasen- und Hämmern synchron dem Herzschlag. Im Juni 1914, also über 1/1/2 Jahre nach Beginn der Erkrankung wurde Patient in die Nervenabteilung der medizinischen Klinik aufgenommen. Seine Beschwerden bestanden damals in zunehmenden Kopfschmerzen in der linken Schläfe und am aufsteigenden Ast des linken Unterkiefers; der Kopf wurde immer in schiefer Zwangshaltung getragen. 
Er hörte auf dem linken Ohr schlechter und es bestand linksseitige Gesichtslähmung, schmerzhaftes Gefühl in der linken Wange. Letztere Beschwerden sollten besser geworden sein; gleichzeitig klagte er über Schmerzen in der linken Schultermuskulatur.

Der am 23. VII. 1914 erhobene objektive Befund ergab Folgendes: Gross gewachsener, kräftiger Mann, in gutem Ernährungszustand; Muskulatur und Fettpolster gut entwickelt. Gewicht $62,0 \mathrm{~kg}$. Haut und Schleimhaut gesund; keine Narben. Zunge auf der linken Seite belegt. Rachenorgane gesund. Am Halse ein an der linken Halsseite hinaufziehender Strang von Daumendicke, an dem einzelne Drüsen palpabel sind. In der linken Fossa supraclavicularis medialwärts ein klein eigrosser Tumor fühlbar. Sonst Thyreoidea ohne Befund.

An den Brust- und Bauchorganen nichts Krankhaftes. Bewegungen der Halswirbelsäule nach allen Richtungen möglich, nach hinten schmerzhaft. Kein Stauchungsschmerz; vom Rachen aus nichts an der Wirbelsäule fühlbar.

Nervensystem: Schädel normal konfiguriert, nirgends klopfempfindlich. Nn. occipitales nicht druckempfindlich. Lidspalten gleichweit. Augenbewegungen frei, geringer Nystagmus nach rechts. Pupillen gleichweit, reagieren prompt auf Licht und Akkomodation. Kornealreflexe beiderseits schwach (links < rechts?).

Augenhintergrund normal.

Es besteht linksseitige Hirnnervenlähmung im Gebiet der Nn. V, VII, VIII, IX, XI, XII.

Hypästhesie der linken Gesichtshälfte und Schwäche im linken Masseter. Der Facialis anf dieser Seite paretisch, am deutlichsten im unteren Ast. Schwerhörigkeit und Vestibularisübererregbarkeit daselbst.

Geschmacksstörung auf der betroffenen Seite.

Gaumensegel wird beiderseits gleichmässig gehoben.

Die linke Schulter hängt etwas herab. Der: ${ }^{*}$. sternocleidomastoideus und trapezius sind links atrophisch und paretisch. Kopf wird schief nach links gehalten. Die linke Zungenhälfte ist atrophisch und zeigt fibrilläre Zuckungen, Zunge weicht beim Vorstrecken nach links ab.

Reflexe an den Extremitäten alle links= rechts vorhanden.

Motilität zeigt normale Verhältnisse. Sensibilität intakt.

Wassermannsche Reaktion im Blute negativ.

Die Psyche zeigte nichts Abnormes. Patient wurde auf Wunsch nach einigen Tagen entlassen.

Anfang Mai 1915 kam Patient wieder; er hatte nach wie vor über Kopfschmerzen zu klagen, alle 14 Tage bis 3 Wochen war Erbrechen aufgetreten und im Februar 1915 beobachtete er, dass sich hinter dem linken $\mathrm{Ohr}$ eine Geschwulst bildete; diese Geschwulst wurde dicker und 3 Wochen vor Wiedereintritt ir die Klinik schmerzhaft; er hörte jetzt auf dem linken Ohr kein Klopfen mehr, aber ein Blasen. Schluckbeschwerden und oft Husten beim Trinken. Angeblich wäre er im Februar 1915 fast erstickt, weil sich Speiseteile vor die Luftröhre gelegt hatten. Die Schmerzen, die periodisch kommen, sitzen jetzt hauptsächlich hinter dem linken Ohr. Sehvermögen wechselnd.

Der im Mai 1915 erhobene objektive Befund ergibt eine Gewichtsabnahme von $14 \mathrm{~kg}$ (48 $\mathrm{kg}$ gegen $62 \mathrm{~kg}$ ). Der Kopf ist aktiv und 
passiv frei beweglich, dabei nicht schmerzhaft. Larynx nach reehts abgedrängt. Der Tumor in der linken Supraclavikulargrube ist immer noch palpabel, er pulsiert nicht. Hinter der linken Ohrmuschel über dem Processus mastoideus pulsierender Tumor von Kleinapfelgrösse, der auf Druck nicht schmerzhaft ist; darüber laut blasendes systolisches Geräusch. Die Pulsation des Tumors wird am Occiput bis über die Mittellinie gefühlt. Der linke Sternocleidomastoideus nicht mehr palpabel; der linke Splenius capitis atrophisch. Beide Karotiden pulsieren lebhaft. Am linken Unterkieferwinkel eine haselnussgrosse Drüse. Die Thoraxperkussion ergibt eine nach oben und links vom Gefässband verbreitete Dämpfung, der auch röntgenologisch eine starke Verschattung nach dem linken

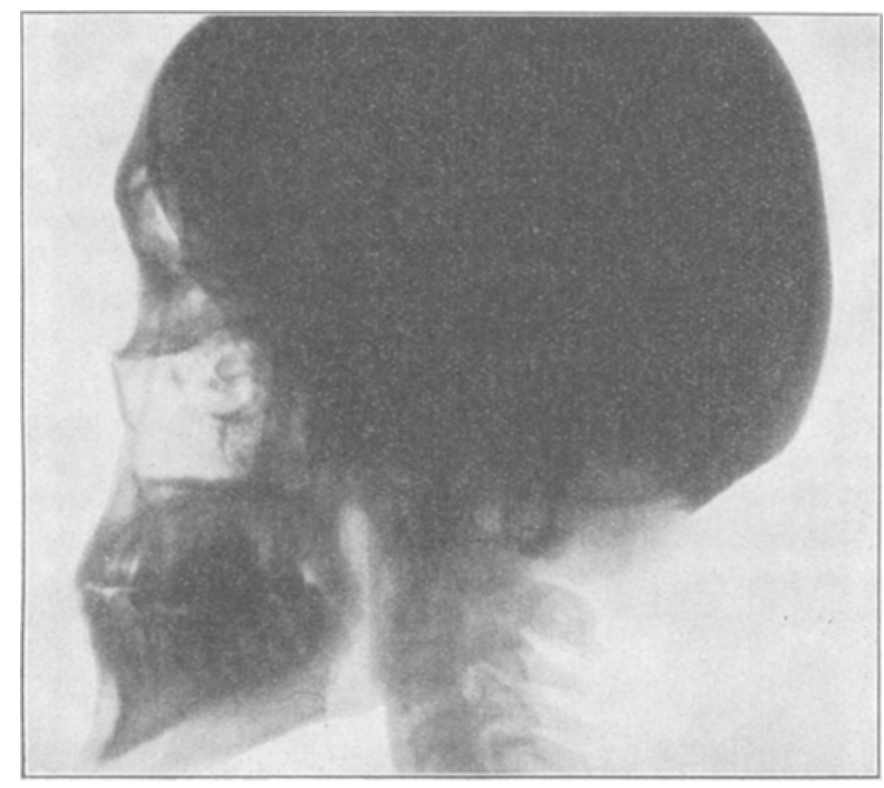

Fig. 1.

Spitzengebiet zu entspricht (Struma substernalis). Herz- und Lungenbefund normal. Blutdruck $120 \mathrm{~mm} \mathrm{Hg}$. Die Pulsation der Aorta abdominalis ist deutlich fühlbar.

Nervensystem: Gehirnnerven rechts durchweg normal innerviert. Auf der linken Seite Erweiterung der Lidspalte, Facialislähmung; Schwäche im Masseter bei intaktem sensiblen T'rigeminus, Taubheit, Geschmackslähmung an der Zungenwurzel, Parese des linken Gaumensegels und Recurrenslähmung. Die Zunge weicht nach links $a b$, ist atrophisch und zeigt fibrilläre Zuckungen auf dieser Seite. Linke Pupille entrundet, die rechte reagiert träger auf Licht wie die linke, beiderseits Papillitis n. optici. Nystagmus horizontalis.

Linker oberster Trapeziusanteil und Sternocleidomastoideus atrophisch paretisch; Splenius geschwunden. Rachenreflex nicht auslösbar. Sehnen- und 
und Periosreflexe der Extremitäten links=rechts: in normaler Stärke auslösbar.

Bauchdeckenreflexe fehlen. Gang schwankend, unsicher. Koordination intakt. Kein Rombergsches Phänomen. Kraft in allen Muskelgruppen ausser in dem atrophischen linken M. sternocleido und im oberen Trapeziusanteil gut. Hautsensibilität intakt für alle Reizqualitäten. Die elektrische Untersuchung ergab keine EaR. im Accessoriusgebiet, nur mässige Herabsetzung der direkten und indirekten Erregbarkeit. Im linken Facialis-

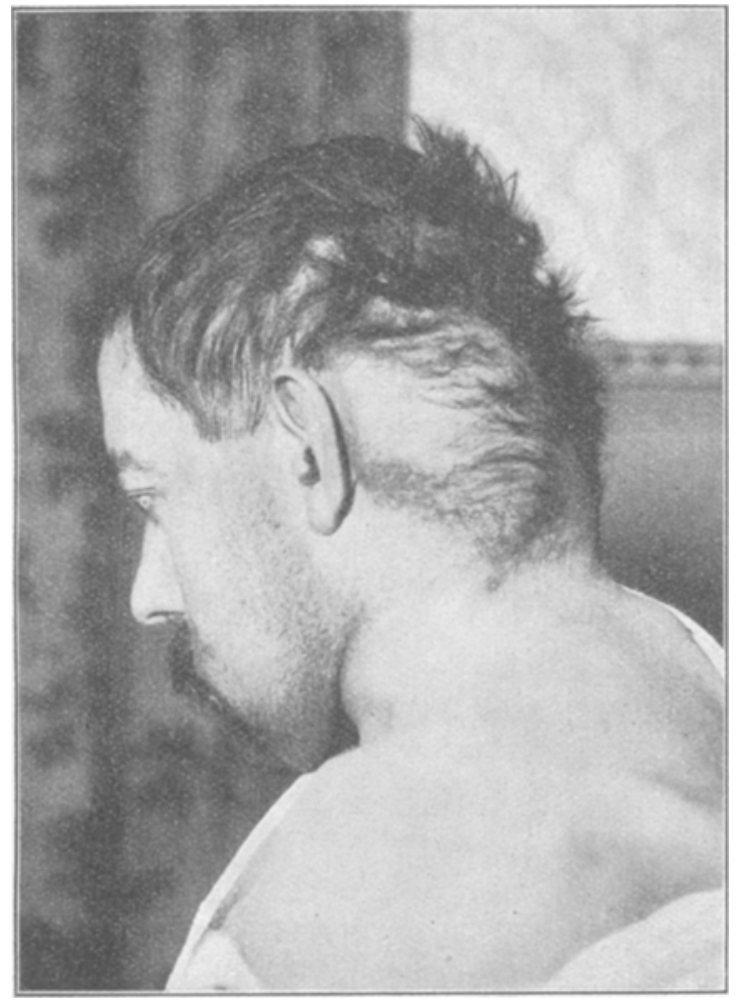

Fig. 2.

gebiet komplete EaR. Der röntgenologische Befund zeigt Arrosion der linken Hälfte der Hinterhauptsschuppe nahe dem Proc. mast. (siehe Abbildung). Der Knochenrand ist unregelmässig ausgezackt, das Foramen der Arteria vertebralis am Atlas erweitert.

Zusammenfassend lässt sich sagen: Ein Mann in mittlerem Lebensalter erkrankte an ausstrahlenden Schmerzen in der Hinterhauptsgegend, die bald von vorübergehender Schluck- und Sprachstörung begleitet waren, es stellte sich in der Folge linksseitige Gesichts- und 
Zungenlähmung und vorübergehende Steifigkeit der Halswirbelsäule ein; auch das Gehör leidet, es tritt mit dem Puls synchrones Blasen und Klopfen im Ohr ein; der linke Sternocleido und oberer Trapezinsanteil atrophieren, der Kopf wird in schiefer Zwangshaltung getragen. Endlich tritt Heiserkeit, Geschmacksstörung, unsicherer Gang, beiderseitige Staungspapille sowie ein pulsierender Tumor am Occiput hinzu.

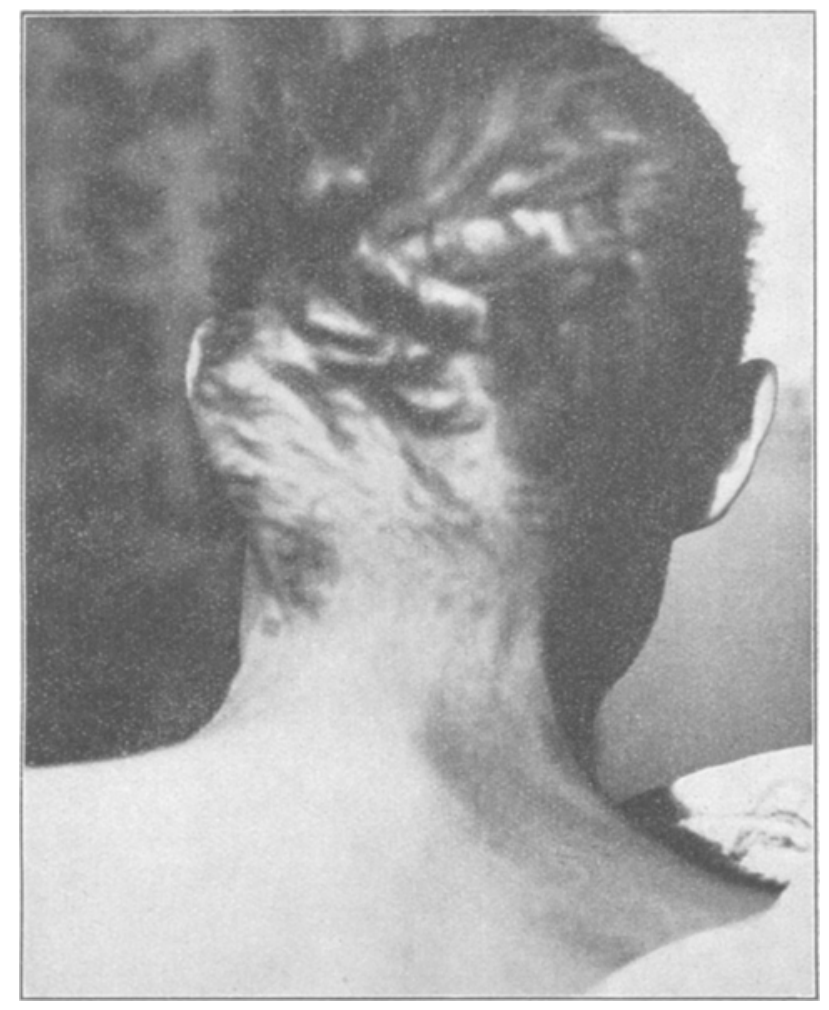

Fig. 3.

Der Sitz des Prozesses stand von Anfang an fest. Die Art desselben blieb dunkel, bis der Nachweis einer pulsierenden Geschwulst mit systolischem Gefässgeräusch bei der zweiten Aufnahme des Kranken auf die Klinik Klarheit brachte. Die Röntgenaufnahme zeigte die durch das Aneurysma bedingte Arrosion des Hinterhauptknochens in recht beträchtlicher Ausdehnung. Infolge Durchbruchs durch das Occiput wurde eine wirksame Dekompression des Gehirns geschaffen. Die Bilder geben über Lage und Grösse des aus der hinteren untẹren 
Schädelhöhle durehgebrochenen Aneurysmateils und über die Ausdehnung der Knochenzerstörung genügend Aufschluss, so dass auf eine eingehende Beschreibung verzichtet werden kann.

Es dürfte dies der einzige bis jetzt bekannte Fall sein, in welchem es zur vollständigen Arrosion der Hinterhauptsschuppe durch ein Aneurysma der Arteria vertebralis gekommen ist.

\section{Literatur.}

1) Griesinger, Arch. f. Heilkunde. Bd. 3. XXIX, 1862. Fortgesetzte Beobachtung von Hirnkrankheiten S. $549 \mathrm{ff}$.

2) Oppe, Ein Fall von Aneurysma der Arteria vertebralis bei einem 7jährigen Knaben. Münch. med. Abh., 2. Reihe, 1892.

3) Möser, D. A. f. klin. Med. XXXV, S. 418.

4) Schultze, Friedr., Virch. Arch. 65. Bd. 3.

5) Oppenheim n. Siemerling, Charité-Annalen. Bd. 12, S. 331.

6) Hoffmann, E. v., Wiener klin. Wochenschr. 1894, S. 824.

Kasuistische Litteratur siehe auch in den Dissertationen von $\mathbf{J}$ ul. Hey (Inaug.-Diss. Berlin 1898) und Hans Krey (Inaug.-Dissert. Greifswald 1891). 